\title{
The Situation of Cancer Treatment in Ethiopia: Challenges and Opportunities
}

\author{
Werissaw Haileselassie ${ }^{1}$, Tefera Mulugeta ${ }^{2}$, Wondemagegnhu Tigeneh ${ }^{3}$, Mirgissa Kaba ${ }^{4}$, Wajana Lako Labisso ${ }^{5}$ \\ 'Department of Reproductive Health and Health Service Management, School of Public Health, ${ }^{2}$ Department of Nursing and Midwifery, School \\ of Allied Health Sciences, ${ }^{3}$ Department of Oncology, School of Medicine, ${ }^{4}$ Department of Preventive Medicine, School of Public Health, ${ }^{5}$ Department \\ of Pathology, School of Medicine, College of Health Sciences, Addis Ababa University, Addis Ababa, Ethiopia
}

\begin{abstract}
Background: Less attention is given to cancer treatment and control in Ethiopia.
Methods: To investigate the challenges and opportunities facing cancer treatment services in Ethiopia. A purposive sampling technique was applied to recruit the study subjects from Black Lion Specialized Hospital Oncology Department. A semi-structured interview guide was used to investigate challenges and opportunities in oncology service in Ethiopia. Data was transcribed and coded by two independent coders and analyzed thematically in reference to the objectives.

Results: Fifteen professionals from four different disciplines were interviewed on opportunities and challenges facing cancer treatment in Ethiopia. Out of these respondents 3 were senior clinical oncologists while 4 individuals were senior oncology residents. The rest were 2 medical Physicists, 2 radiotherapy technologists and 4 oncology nurses. Majority (80\%) of the respondents were males. We demonstrate that the challenges of cancer treatment service in Ethiopia emanate from the patients themselves, the administrating body, the professionals, and the technology limitations. In general, the result of this study was grouped under the following five themes: Customer-related challenges, provider-related challenges, facility-related challenges, technology-related challenges and the opportunities. Conclusions: Several assignments are waiting for the policy makers, the professionals, the communities and other concerned bodies to combat the alarmingly growing burden of cancer in Ethiopia. Escalating the awareness of the general population about cancer, expanding well-developed diagnostic and treatment centers, and producing well-trained competent oncology professionals are the forefront challenges in combating cancer in Ethiopia.
\end{abstract}

(J Cancer Prev 2019;24:33-42)

Key Words: Cancer, Ethiopia, Challenges, Opportunities, Black Lion Specialized Hospital

\section{INTRODUCTION}

Cancer is a major public health problem in many low income countries (LICs), including Ethiopia. A survey from Addis Ababa cancer registry indicates that 64,000 new cases of cancer occur annually [1]. The type of cancer treatment modality that is applied for particular patient depends on the type and stage of the cancer and the availability of the proposed treatment modality [2]. The classical cancer treatment modalities are surgery, chemotherapy, hormonal and radiation therapy. More advanced treatment modalities are bone marrow transplantation, targeted therapy, personalized therapy, gene therapy, intensity modulated radiotherapy, image-guided radiation therapy. The latter are fancy cancer treatment modalities that are not easily available in low and middle income countries (LIMCs); however, they are characterized by low side effects and effective outcomes [2,3].

The treatment outcome of cancer patients depends on stage and types of the cancer, biology of tumor and patient factors. Accordingly, the ultimate goal of cancer treatment is to relief the pain and search for cure for the patient. To this end, the field is facing several challenges. The common challenges in the arena of cancer treatment is the steadily rising cost of the chemotherapy,

Received February 7, 2019, Revised March 21, 2019, Accepted March 21, 2019

Correspondence to: Wajana Lako Labisso

E-mail: wajana.lako@aau.edu.et, ORCID: Wajana Lako Labisso, https://orcid.org/0000-0003-0687-3745

Copyright (C) 2019 Korean Society of Cancer Prevention

(c) This is an Open Access article distributed under the terms of the Creative Commons Attribution Non-Commercial License (http://creativecommons.org/licenses/by-nc/4.0) which permits unrestricted non-commercial use, distribution, and reproduction in any medium, provided the original work is properly cited. 
in-accessibility and unaffordable treatment modalities, drug resistance of cancer cells, complexity and expensiveness of the radiotherapy equipments and centers. These are more pronounced and complex problems for patients and clinicians in LMICs [3-5]. The common challenges in patient sides are lack of awareness of the signs and symptoms of cancer, shortage of traveling cost to hospital and financial deficiency for diagnosis and treatment costs, which thus leads many to seek traditional treatments instead [6-10]. Shortage of well-trained oncologists, specialized cancer nurses and medical physicist are additional bottlenecks of fighting cancer in these countries [11,12]. The radiotherapy machines and centers are not proportional with the growing population and rising burden of cancer in LMICs [12]. According to the International Atomic Energy Agency (IAEA), whilst developed countries usually have one radiotherapy machine per 250,000 people, most LMICs often have only one machine per seven million. In some serious cases one machine is for more than tens of millions of people. More regrettably, tens of countries completely lack access to any radiotherapy treatment facilities $[13,14]$.

Ethiopia is the second populous country in Africa with estimated 100 million people. The alarmingly growing population together with lifestyle changes will designate rising liability of cancer. However, oncology services are wholly inadequate and the country is not well-prepared to bear the growing burden of cancer. It has only one fully functioning public radiotherapy center, with a handful of doctors and nurses, struggling to serve the entire country. Moreover, national cancer registry is lacking though Addis Ababa cancer registry has been established in 2013 [15].

Cancer is a major cause of morbidity and mortality globally and more than 10 million new cases and over 7 million deaths occurred in 2000 alone. In the same year, significant number of cases and deaths were contributed by LMICs [16]. A survey from Addis Ababa cancer registry indicates that 64,000 new cases of cancer occur annually among which cervical and breast cancer constitute the significant majority. Mortality from cervical cancer alone was estimated to be 3,235 in Ethiopia [17]. Therefore, cancer is now becoming the major health problem in Ethiopia; however lacking equivalent attention. The routine activity of cancer treatment service center in Black Lion Specialized Hospital (BLSH) is impaired by several factors that are not assessed scientifically so far. Therefore, the objective of this study was to explore the challenges and opportunities facing cancer treatment in Ethiopia.

\section{MATERIALS AND METHODS}

\section{Study setting and study participants}

The study was conducted in August 2018 at BLSH where the sole radiotherapy referral center in Ethiopia. The hospital is a tertiary level teaching hospital affiliated with the college of health sciences of Addis Ababa University. A hierarchy of the organization and the place where the study participants were selected was indicated in figure and flow chart (Fig. 1 and 2). The study participants were practitioners working in the oncology department of BLSH. A total of 15 practitioners were included in this study. The sample size was determined in such a way that rich data can be obtained from the study participants. Emphasis was given to include the diverse views of the different categories of professionals. To minimize the bias, attention was given to assess the perspectives of majority of the individuals in each category of profession. They were experienced oncologists, oncology nurses, medical physicists, radiotherapy technicians and senior residents who were working at BLSH oncology clinic from 2000 to 2018. So far, there are only five oncologists in the country of which three oncologists are currently working in the oncology center and all the three oncologists were included in the study. Moreover, four senior oncology residents were selected for the interview. Besides two medical physicists, two radiotherapy technologists and four nurses were also involved in the study. Purposive sampling technique was applied to select the study participants.

\section{Method of data collection}

Qualitative study using in-depth interview was conducted to explore challenges and opportunities in relation to cancer

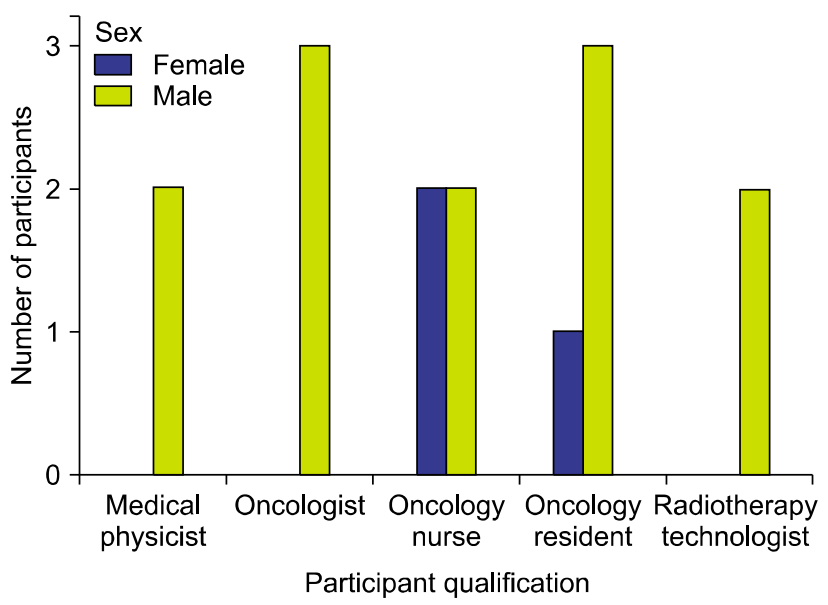

Figure 1. Participant qualification by sex of the study subjects. 


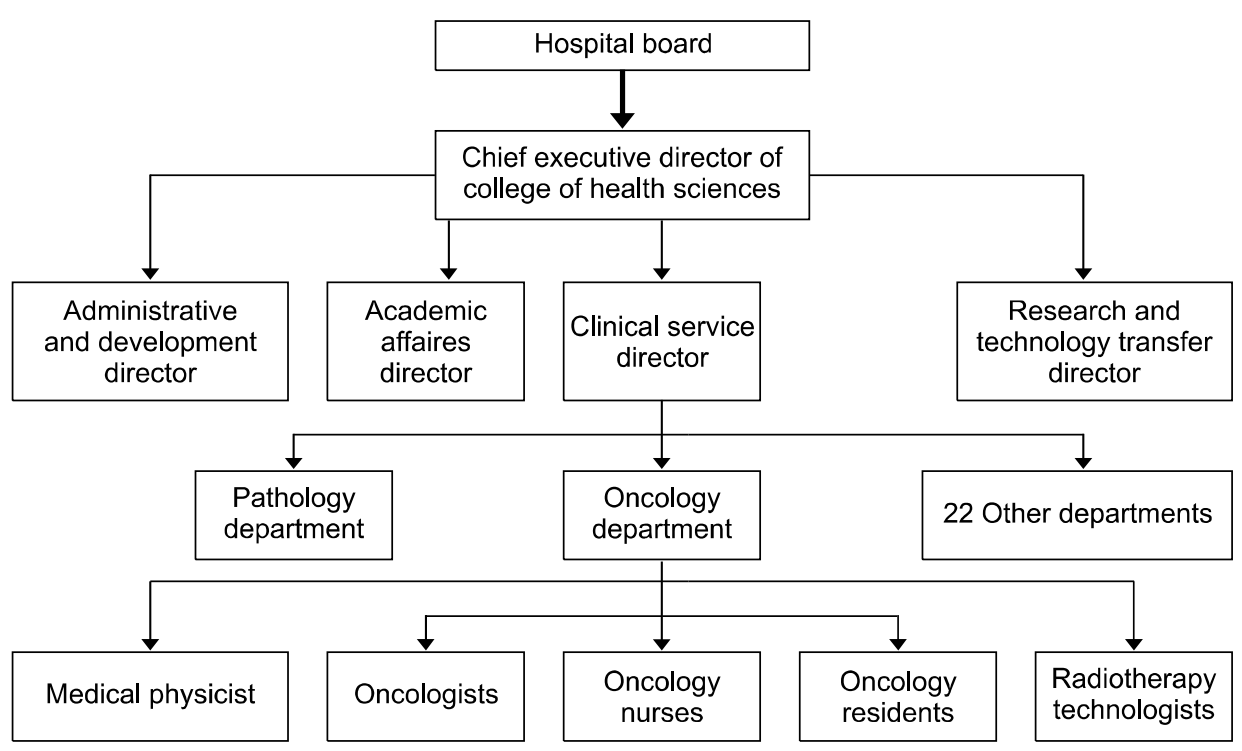

Figure 2. A flow chart depicting a hierarchy of the organization and the places where the study participants were selected. treatment in Ethiopia. Data was collected using semi-structured interview guide which was developed focusing on challenges, solution attempted, opportunities and strategies to improve cancer treatment in the oncology and based on the objective of the study. The interview tool was reviewed and substantiated by the research team before it is used for data collection to ensure it helps to answer study questions. Interview was audio-taped following permission from participants. The interview was conducted by an experienced investigator in collaboration with research assistant using interview guides at BLSH in dedicated rooms where participants can comfortably discuss with data collectors. Details were elicited through probing. Each interview took an average of 45 minutes.

\section{Ethical clearance}

After a brief explanation about the aim of the research and the confidentiality of the information collected from the study participants, verbal consent was obtained from participants.

\section{Data analysis}

Following transcription, the investigators thoroughly read the raw data. Following this we coded the transcribed data in light of the objectives of the research. The data was transcribed and coded by two independent coders that helped to define themes in reference to the objectives. Then, inter-coder verification was done to ensure that the codes were the reflection of the collected data. Codes were further categorized under themes and variations between the independent coders were discussed to reach at common themes. The themes were reviewed for validity, relevance, and correct representativeness of what was contained within the data. At the end, themes were defined as client-related challenges, facility-related challenges, profession-related challenges, technology-related challenges, and opportunities in cancer treatment were identified as themes. A thematic latent analysis with inductive approach was employed to analyze the data.

\section{RESULTS}

Totally, $86.7 \%$ of the respondents were males while the rest were females (Fig. 1). The finding of this study was presented under the following five themes: patient-related challenges, facility-related challenges, health care provider-related challenges, technology-related challenges, and opportunities and perspectives in cancer treatment.

\section{Perceived patient-related challenges}

The study participants mentioned that it has been a common phenomenon to see tired patients and their families gathered tightly together in dark stairwells, or benches made up of wooden-linings the hallways outside the oncology ward with dim light. Despite the dramatic rise in the number of cancer cases, the patients had very limited knowledge about the cause as well as the signs and symptoms of the disease. Moreover, they perceived that patients who developed cancer have no hope of cure and consider it as death sentence. That is why most cancer patients were suffered from stress and anxiety. Even if one wishes to have treatment he/she must wait for 5 to 6 months in average. Moreover, they showed the tendency to give priority to traditional medicine and religious centers. The study participants stressed in the awareness gap responding: 
"Cancer patients have poor awareness on the disease. Sometimes they associate cancer with God's punishment or curse. For instance, when you tell to the patient that he/she has cancer, they consider it as taboo i.e., they feel as if they will be discriminated by the society. And they immediately look for holy-water or traditional medicine. They seek orthodox medical care after attempting all these means of treatment. As a result, they come to our cancer treatment center after the cancer has metastasized and passed its curable stage (RO-1, RR-2)".

According to the study participants, cancer patients avoid modern medicine believing that spiritual solutions are more effective than modern medicine/chemo and surgical therapy. Some other patients desired to go to traditional healers because traditional medicine gives them immediate but temporary symptomatic pain relief from the disease.

Patients came to the hospital selling their family assets such as livestock and even home to cover the trip and treatment costs even though there is no assurance of cure. This puts the family in desperate situation. According to the interview respondents the service cost at BLSH is fair since the service is subsidized by the government. And even for patients who can bring official letter from their local administrative unit permitting free service, the hospital gives the service free of charge. However, the study participants reported that the service is not persistently available at the hospital and sometimes there is interruption. One of the study participants emphasized:

"People go home to die since there is a long waiting list and chronic chemotherapy drugs shortage because of the imbalance between supply and demand. When patients want to buy the drugs from private pharmacies, the cost is totally unaffordable and sometimes the private pharmacies unacceptably increase the cost of the drugs if they know that the drugs are not available at the BLSH" (RN-1).

It was pointed out by one of the study participants that cancer patients and their relatives had negative attitude towards cancer treatment modalities, particularly for radiotherapy. They believed that radiotherapy damages the internal organs of the human body. One of the respondents astonishingly mentioned that:

"Cancer patients believe that radiation therapy burns human body. They thought that a person treated with radiation end up with death. When they consult the family or neighbors whether to take a prescribed radiation therapy or not, they were not encouraged to go to the treatment center and take the treatment regimen. Consequently, it is with dread that they come to the treatment center" (RR-2).

In addition, the respondents reported that women cancer patients did not know about self-breast examination and ignore lumps. They sought treatment at advanced stage of the disease.

\section{Service burden and professional-related challenges}

Every day approximately 35 to 50 and 65 to 70 patients get chemotherapy and radiotherapy services in oncology unit, respectively. Close to 5,500 new cancer cases were seen in the treatment center per year. According to data from the hospital's oncology unit, more than 500 adult and pediatric cases with hematologic malignancies were seen in the hematology clinics every year. Many patients with cancer were also seen at the surgical, gastrointestinal and gynecology clinics. The most common adult cancers are cervical, breast, sarcomas, head and neck, and colorectal cancers, while leukemia, lymphoma, retinoblastoma and osteosarcoma constitute the bulk of pediatric cancers. Currently, the center is giving clinical oncology training for physicians and nurses. Besides, it has finalized its preparation to launch new undergraduate programs in medical physics and radiotherapy technology. There is a recently inaugurated satellite comprehensive cancer treatment center in Addis Ababa in Woreda Eight Health Center on 6 February 2016.

Despite this huge burden, there are currently few senior trained oncologists in Ethiopia. Of which only three are employed at BLSH to serve a population of more than 100 million. The participants mentioned the fact that there is shortage of trained manpower. They reported that they are stretched to meet the demands of cancer patients waiting up to receive treatment. This led to large work load which can be an impediment to quality oncology service. The respondents pinpointed the fact that the situation is not peculiar to oncologists but also to the other disciplines, such as oncology nurse, radiotherapy technologists and medical physicists. As a result, all practitioners were overwhelmed with high number of patients who came from different corners of the country. Taking this dire circumstance into consideration, the department launched oncology residency program before five years. This year 4 additional fresh graduate oncologists joined the battle. There is also an oncology nurse training program in conjunction with oncology residency program. While the curriculum for radiotherapy technologist has been finalized to launch out the training, the curriculum for 
medical physicists is underway. One of the respondents revealed:

"There is lack of adequate number of trained oncologists, oncology nurses, radiotherapy technologists and medical physicists despite the escalated number of cancer cases. This is one big challenge to combat cancer in Ethiopia" (RO-3).

There is poor training and development for radiotherapy technologists. They reported that they had received few short term training. Others indicated the very fact that they had been exposed to some domestic as well as international training on cancer care. The practitioners felt that they need to get training on modern cancer treatment technologies. They boldly remarked that absence of specialized training is putting its impact on skill development, passion and self-reliance in rendering quality comprehensive cancer care service. The need for capacity building to deliver quality cancer service is indispensable. This is evidenced from one of the respondents:

"I didn't get adequate training as far as my area of specialization is concerned. There were few short term trainings which did not enhance my skill. Trainings should be satisfactory in terms of bringing desired capacity building. I think there should be circumstances which help win the commitment of employee" (RR-2).

Furthermore, the participants stressed the importance of creating an environment where they can exercise what they have been trained for improved cancer care. They said that for the training to be fruitful, important arrangements must be made to see the training outcome and improve the service. Regarding continuity of health care team, there was no frequent change of team of health care workers. One of the respondents said,

"I had taken training on linear accelerators (LINAC), the devices most commonly used for external beam radiation treatments for patients with cancer, many years ago. However, because of the absence of the device, I cannot work with the device and forgot what I had been trained" (RR-1).

Despite all the aforementioned challenges, the participants explained their readiness to provide timely service to cancer patients. They said that they are highly committed to help and guide cancer patients but serious consideration should be taken in to account in order to improve cancer service in Ethiopia. They mentioned that they had gone to the extent of preparing booklet on cancer treatment using working language of the country (Amharic) to raise the awareness of patients.

\section{Facility-related challenges}

The study participants responded that many more are waiting for their turn since there is chronic shortage of space and medical equipment and materials. The participants mentioned that there were only three outpatient departments (OPD). The two OPDs were made from a single house through partitioning using play wood. This had compromised not only the oncology service but also the training of the already launched programs (oncology residence and oncology nurse). The participants attributed the presence of dozens of frail cancer patients and their relatives huddled together in stairwells and around the oncology ward with the embellished shortage of space. Sadly a respondent emphasized that:

"You commonly see patients surrounded by their families around the oncology ward waiting for their turn. They don't want to wait for long time since come to the cancer center traveling long distances from different parts of the country and the cost of travel and, living and accommodation in Addis Ababa is extremely high. At times the patients found this desperate condition, it is unbearable for them and they get in quarrel with the service provider complaining the long waiting time" (RN-1, RN-2).

Another big challenge identified by the respondents was the absence of combine clinic to provide integrated and comprehensive cancer care. They mentioned that this impaired the cross-talk to exchange clinical information impacting the treatment outcome since cancer treatment service is a team work. One respondent reiterated that:

"Oncology service requires a pathologist who can diagnose using recommended investigations, a radiologist who can stage the disease and a surgeon who can render surgical therapy beside the oncologist for comprehensive cancer care. We need combined clinic which can bring practitioners together for concerted efforts to avert over or under treatment. The absence of combined clinic leads to disagreement regarding treatment plan for the patient. Recently we have started combined clinic on breast cancer and colorectal cancer" (RO-3).

Lack of adequate bed and chronic shortage of chemotherapeutic agents were the other challenges reported by the participants. They emphasized that shortage of bed hindered the on time 
implementation of treatment plan for the patient leading to late initiation of treatment for the disease after it has escaped beyond it can be reversed. At times it was mentioned that the oncology center interrupts chemotherapy service giving it fragmented nature. The participants said that patients will be forced to buy drugs from private pharmacies at burdensome cost. The participants described the unacceptably high cost of drugs in the outside market as inhuman. As a result, it was gathered that this worsens the dire situation associated with extended waiting and turnaround time as well as lack of infrastructure. One respondent stated that:

"It is very outrageous to hear unfairly high cost of cancer drugs in private pharmacies in view of the overwhelming lack of infrastructure and shortage of basic services. It is totally inhuman. We have to be a voice for weary cancer patients struggling with stress and hopelessness beside the disease. For instance a one to two birr (Ethiopian currency) plasil (metoclopramide) costs 20-30 birr in private pharmacies. The pharmacies unacceptably raise the cost if they know that the drugs are not available at BLSH" (RN-2).

The presence of only one radiotherapy centre for the population of more than 90 million was mentioned as one challenge by the study participants. Evidences obtained from sculpture show that the radiotherapy center was built in October 14, 1997 by the Ethiopian Government and IAEA. It has the only functional radiation machine for the entire country requiring patients to wait up to six months and more for treatment of their diagnosis. It has been reported that at times the service interrupts for months owing to machine outage and poor maintenance efficiency. This further extends the waiting time thereby prolonging suffering time and accelerates the journey to death. Consequently, the effort to restore health, hope and life to patients and their families becomes cumbersome.

\section{Technology-related challenges}

It was reported that computerized data management system is not in place at BLSH oncology center. Participants considered the situation as an obstacle for the centers aspiration to become center of excellence in cancer diagnosis, treatment, care and research. Moreover, they mentioned that there is fragmented information and communications technology (ICT) service which can be an impediment for teleconference and oncology training. They also reflected their fear of loss of patient records due to the absence of well-equipped data manager and dependable data management system. This was taken as a threat for establishing reliable cancer registry.

\section{Opportunities}

A complex crosstalk exists among the challenges and the challenges are a window of opportunities (Fig. 3) for improvement of the oncology services. BLSH as a big academic and referral center was regarded as main opportunity to ensure the aspiration to become center of excellence in cancer care and realize normalized patient's quality of life and diminished early deaths. The current aspiration and commitment of the government to strengthen BLSH and expand cancer treatment centers to other parts of the country was taken as good opportunity in view of the growing burden of cancer. Telecom service expansion was viewed positively to improve cancer treatment service along with the oncology training and research. The participants were optimistic towards the increasing number of interested groups and campaign on cancer. The challenges described in this study are strongly interlinked and influencing each other. A new oncology service center is constructed by BLSH

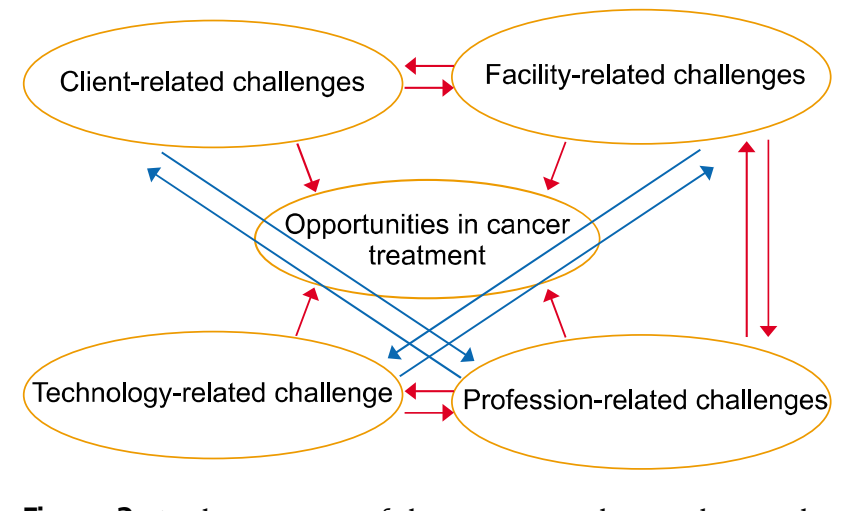

Figure 3. A schematic view of thematic map indicating the complex interaction among the challenges and links between challenges and opportunity in cancer treatment. A well-aware client demands quality services and plays its own role in acquiring good oncology service facility and the later determines the satisfaction of the former. A dedicated and well-trained professional can positively influence the attitude of the clients towards the oncology services. The professionals have also a responsibility of introducing new facilities for oncology service by convincing and working together with the authorities. In addition, the facilities have direct impact on the professional satisfaction, effectiveness of the experts and the quality of the service. The conduciveness of the working-environment and the availability of functional instruments and materials needed for service delivery affect the daily activity and motivation of the experts. Medical technology is dynamically changing; thus, the professional is supposed to update oneself with everyday changing technology and should be able to utilize the cutting-edge technologies to provide satisfactory services. The technology should be also part of the facility and the later should encompass the former. 
jointly with the Ministry of Health. It provides comprehensive cancer treatment services including surgery, radiation therapy and chemotherapy. The center has the capacity to provide diagnosis and treatment services for up to 85 patients per day. Thus, it eases the huge burden on BLSH thereby increasing access to cancer treatment for patients. Therefore, respondents hope that the future will be bright with all collaborating stakeholders.

\section{Solutions attempted}

The oncology department of BLSH has tried to expand cancer treatment service to a health center located in Lideta sub-city which is received from region 14 health office. The center is providing chemotherapy in ward and at out-patient. It is giving the service for both adults and pediatric age groups. It also has the capacity to provide diagnosis and treatment services to about 85 patients daily. As such it eases the huge burden on BLSH thereby increasing access to cancer treatment. Moreover, procurement of LINAC was completed.

\section{DISCUSSION}

Cancer has not been considered as a health priority in LICs as the countries are still battling with infectious diseases, such as tuberculosis, malaria and human immunodeficiency virus/acquired immune deficiency syndrome among others [16-18]. The inevitable truth is that the countries are suffering from the alarmingly growing burden of cancer. Most of the cancer deaths in LICs are preventable if it had been detected earlier and appropriate treatment means are applied [19-21]. One of the limiting factors of the outcome of cancer patients is the stage of the disease at which patients are presented to the hospital. Noticeably, most of cancer cases in Ethiopia are presented at advanced stage of the disease. This is mostly due to the fact that cancer patients and the general population in Ethiopia have very poor awareness about cancer [22-24]. As the cancer is detected in late stage, patients are recommended for invasive therapeutic modalities, which can put additional psychosocial pressure on the patients and their families. Due to long waiting list for radiotherapy and unaffordable expense of chemotherapy, patients and family members panic when diagnosed with cancer. Families and patients are often challenged with impractical dilemmas when making the decision as to whether they will follow the therapy. Therefore, the impact of cancer detection in Ethiopian patient, as in other African patients, is multiple. This is because there is strong family attachment and economical and psychosocial interdependency within Ethiopian family members. The financial impact is much worse in Ethiopian and other LICs' patients than the developed countries' patients, because there is no well-established insurance system in these countries. If the patient is the main source of income in the family, then the family uses all the possible options to afford pay for treatment. If the patient is a mother, then the influence is much worse and stronger psycho-sociological impact is expected on the family. In such cases, the children will be left alone because the father will be taking care of his wife. In addition, either of the cancer treatment means might financially cripple the family and prevent them from being able to feed their healthy children [25].

There is unavailability of affordable treatment means in LICs, which in turn, leads to high rates of treatment abandonment. Patients usually instead seek alternative or traditional treatments $[26,27]$. Some even consider that radiotherapy can burn the body, indicating urgent public education concerning cancer treatment. This is common misconception in several parts of other African patients where people refused radiotherapy and cancer chemotherapy [28-30]. In addition, some of the cancer patients are presented at advanced stage of the disease where noninvasive curable therapeutic approach is impossible. This results in complications and premature death. Furthermore, when patients from low-income backgrounds pursue treatment, it often results in the accumulation of considerable debts in the family, putting significant financial burden on the whole family [25,30].

Mortality from cancer is significantly decreasing in high income countries. This is due to increased awareness of the general population about cancer, availability of advanced diagnostic and treatment means, existence of well-trained professionals in the field and availability of screening programs for particular types of cancers like breast and cervical cancers [31,32]. This is in contrast to our study where the cancer patients have very poor awareness about screening programs, particularly breast cancer and cervical cancers. As a result, significant portion of cancer morbidity and mortality are due to these cancers. This is in line with findings from other LICs where educated and non-educated groups of people are reluctant and do have very poor awareness about the existing screening programs [6,33,34]. In addition, the health system in LICs is not well-prepared to handle the outcome of the cancer screening. Thus, expanding cancer treatment centers, strengthening the capacity of human resource and making available the treatment modality should be the prerequisite to launching screening programs in these regions [25,34].

BLSH oncology department is overwhelmed with persistently 
increasing burden of cancer from all corners of the country. Currently, as the study participants reported, there could be more than 150,000 cancer cases in Ethiopia every year. Oncology practitioners also estimate that there were 150 to 200 patients seen at the oncology department per day. This is unbearable load on the oncologists and other professionals in the department compared to the limited number of human resources in this department. However, it is noteworthy to mention the effort of the hospital and the department in combating the challenges. Launching of oncology residency program, training in oncology nurse and radiation technologist are some of the most important critical measures taken by the department. It is only in such a way that the demand of more than 90 million people can be fulfilled.

Most of other African countries also focus on cancer control by expanding training and strengthening the capacity. Unfortunately, the cancer treatment facilities in Africa are extremely below the standard compared to other developed countries, like United Kingdom where there are 18 dedicated cancer research centres and 34 cancer networks. In addition, the population in United Kingdom has free access to healthcare and the most up-to-date treatment modalities along with access to all types of cancer specialists within the system. In contrary, more than 20 countries in Africa do not have even a single facility with working radiotherapy machines, even though about $63 \%$ of cancer patients need radiation. About $60 \%$ of the radiotherapy machines are found in limited countries, namely South Africa, Egypt, and Morocco. Uganda has one radiotherapy treatment unit. Namibia has two radiotherapy treatment units, which is better than others since it has low population. Ghana has six oncologists and two cancer treatment centers to treat a population of 23 million. This is in contrast to Ethiopia where there is only one radiotherapy unit with one full functioning cobalt 60 machine for more than 90 million people. This indicates that the country has a lot of home works to control rising burden of cancer. Otherwise, the consequence of cancer, if it resumes in the present way, will be beyond health issue [35,36]. Of course, the current proposal of the government to establish five additional new radiotherapy centers in different parts of the country is appreciable. But it needs to be urgently materialized.

In addition, there is limited number of professionals with superficial training. This is in line with several other countries in Africa and beyond [34]. The study respondents indicated that they have limited number of medical physicist, which had great impact in the struggle for cancer control. Cancer treatment will not be effective if the oncologist is not competent enough to manage the cancer with appropriate therapeutic. Several studies indicated that the presence of adequate and competent professionals in the field is directly linked with successful, comprehensive, compassionate and respectful cancer care $[11,25,34,35]$. Therefore, the importance of an adequate and appropriate training for oncology professionals for controlling cancer is inevitable.

Apart from the work burden and limited capacity of the professionals, the conduciveness of the working environment is another critical issue raised in the discussion. According to the study participants tens of patients and family members were sitting and lying on the wooden bench from dawn to dusk. The management of cancer patients was impaired by limited number of beds to start chemotherapy; therefore, the patient should wait for the turn till the bed is free. The same is true in other African countries where the oncology patients wait everyday sitting and lying on extremely uncomfortable environment for long period of time till treatment is initiated. This is in contrary to cancer therapy centers in developed countries with well-established health insurance system. In these countries, people do have easy access to cancer therapy centers and the health care system has full responsibility and accountability in the fate of cancer patients [34-37].

Another critical obstacle in effective and successful cancer treatment is the absence of formal cross-talk among oncologists, pathologists, radiologists, and surgeons. This is an easy problem that does not need any expenses but willing and cooperative minds. In developed countries, there is regular communications among professionals from different fields in order to provide the patient successful and trustful services [34,38]. In previous study, we showed that pathologists were also in need of professional communication with clinicians for better outcome of cancer patient managements [28]. Unfortunately, this kind of communication is poor in many LICs cancer managing professionals [28,39]. It could be due to the high burden of routine daily activities that the practitioners could not set time for formal communication. However, it is very important to reconsider the importance of the communication and initiate it as soon as possible. Studies from other African countries and United Kingdom also indicated that this kind of comprehensive cancer care system had strong impact on the fate of the cancer patients. It improved patient follow-ups and the chance of cure for the patient is very high [34,40].

Several points were identified as a reason for delay in timely implementation of treatment plan for the already belated patient. Most of the reasons however are attributed to extremely limited resources. It was reported in other low settings also that 
shortage of resources played significant role in delivery cancer management services. This could be due to one of the following several reasons: 1) shortage of supply, 2) lack of commitment by the authorities, and 3) economical constraints-unfairly exaggerated cost $[4,6,7,27,34]$

BLSH cancer treatment center is aspiring to be center of excellence in cancer diagnosis, treatment, care and research. Thus, it needs to have well-equipped ICT facility with dedicated and competent professionals in the field. In the contemporary era of cooperative cancer care, ICT system plays great role in facilitating teleconference, telemedicine, and online trainings [41]. This challenge was pointed out in previous study where pathologists considered limitation of information communication technology as a crucial obstacle in practicing telepathology [27]. This report is in agreement with findings in other African countries where poor ICT influenced negatively the success of cancer management services. Aspiring for national cancer registry and screening program could not be achieved in absence of well-equipped data manager and dependable cancer data management system in the country [42-45].

This study indicated that there are several windows of opportunists in an ocean of challenges facing cancer control in Ethiopia. BLSH, as a big academic and referral center, the increasing effort of the regional and federal government to combat cancer, ICT expansion and professional collaborations in trainings and research are some of the common window of opportunities through which brighter future can be aspired. Several state of the art instruments and new building are allocated to oncology department by the hospital. Thus, concerted effort is needed from all concerned bodies to make fruitful all the dreams and plans at hand in order to win the challenge facing cancer treatment in this country.

To our knowledge, this is the first study of its kinds indicating the challenges and opportunity the cancer treatment situation is facing in Ethiopia. Several forms of cancers are preventable if we escalate the awareness of people, screening programs, advanced diagnostic and treatment means in the country. However several assignments are waiting for the government bodies, the professionals, the communities and other concerned bodies to combat the alarmingly growing burden of cancer. Some of the most important challenges mentioned in this study are scaling up the awareness of cancer in the general population, expanding well-developed diagnostic and treatment centers, and producing well-trained competent oncology professionals. As most of the cancer cases are presented at advanced stage of the disease, introducing the palliative care services for cancer patients is critically important. Above all, it is essential to redefine the role of health policy in fighting against cancer; because the inevitable truth is that many citizens are suffering from the alarmingly growing burden of the disease.

One of the limitations of this study was that the sample size is limited because we had limited number of subjects in the field. As a result gender bias was also inevitable.

\section{ACKNOWLEDGMENTS}

The authors thank colleagues who assisted during data collection. The authors are also grateful to the oncology department, oncologists, nurses, medical physicists, and residents who participated in the interview.

\section{CONFLICTS OF INTEREST}

No potential conflicts of interest were disclosed.

\section{REFERENCES}

1. Tigeneh W, Molla A, Abreha A, Assefa M. Pattern of cancer in Tikur Anbessa Specialized Hospital Oncology Center in Ethiopia from 1998 to 2010. Int J Cancer Res Mol Mech 2015:1.1. doi: 10.16966/2381-3318.103.

2. Gerber DE. Targeted therapies: a new generation of cancer treatments. Am Fam Physician 2008;77:311-9.

3. Kawakami K, Nakajima O, Morishita R, Nagai R. Targeted anticancer immunotoxins and cytotoxic agents with direct killing moieties. ScientificWorldJournal 2006;6:781-90.

4. Farmer P, Frenk J, Knaul FM, Shulman LN, Alleyne G, Armstrong L, et al. Expansion of cancer care and control in countries of low and middle income: a call to action. Lancet 2010;376:1186-93.

5. Plesnicar S, Plesnicar A. Cancer: a reality in the emerging world. Semin Oncol 2001;28:210- 6 .

6. Wong LP, Wong YL, Low WY, Khoo EM, Shuib R. Cervical cancer screening attitudes and beliefs of Malaysian women who have never had a pap smear: a qualitative study. Int J Behav Med 2008; 15:289-92.

7. Arslan AA, Formenti SC. Mammography in developing countries: the risks associated with globalizing the experiences of the Western world. Nat Clin Pract Oncol 2009;6:136-7.

8. Rizwan MM, Saadullah M. Lack of awareness about breast cancer and its screening in developing countries. Indian J Cancer 2009:46:252-3.

9. Shyyan R, Masood S, Badwe RA, Errico KM, Liberman L, Ozmen $\mathrm{V}$, et al. Breast cancer in limited-resource countries: diagnosis and pathology. Breast J 2006;12 Suppl 1:S27-37.

10. Urasa M, Darj E. Knowledge of cervical cancer and screening practices of nurses at a regional hospital in Tanzania. Afr Health Sci 2011;11:48-57.

11. Beaulieu N, Bloom D, Bloom R, Stein R. Breakaway: the global 
burden of cancer-challenges and opportunities: a report from the Economist Intelligence Unit. http://graphics.eiu.com/upload/eb/EIU_LIVESTRONG_Global_Cancer_Burden.pdf. Accessed July $27,2010$.

12. Ngoma T. World Health Organization cancer priorities in developing countries. Ann Oncol 2006;17 Suppl 8:viii9-14.

13. Barton MB, Frommer M, Shafiq J. Role of radiotherapy in cancer control in low-income and middle-income countries. Lancet Oncol 2006; 7:584-95.

14. International Automic Energy Agency (AIEA). Setting up a radiotherapy programme: clinical, medical physics, radiation protection and safety aspects. Vienna, IAEA, 2008.

15. African Cancer Registry Network. http://afcrn.org/membership/members/100-Addisababa. Accessed January 14, 2019.

16. Ferlay J, Soerjomataram I, Ervik M, Dikshit R, Eser S, Mathers C, et al. GLOBOCAN 2012 v1.0, Cancer incidence and mortality worldwide: IARC CancerBase No.11. Lyon, International Agency for Research on Cancer, 2008.

17. World Health Organization. United Nations high-level meeting on noncommunicable disease prevention and control. https:// www.who.int/nmh/events/un_ncd_summit2011/en/. Accessed January 10, 2019.

18. Jenkin B. Cancer Control. Non-communicable diseases in the developing world. http://www.cancercontrol.info/cc2014/jenkin/. Accessed January 10, 2019.

19. Sloan FA, Gelband H. Cancer control opportunities in low- and middle-income countries. Washington, DC, National Academies Press, 2007.

20. Ezzati M, Riboli E. Can noncommunicable diseases be prevented? Lessons from studies of populations and individuals. Science 2012;337:1482-7.

21. Anand P, Kunnumakkara AB, Sundaram C, Harikumar KB, Tharakan ST, Lai OS, et al. Cancer is a preventable disease that requires major lifestyle changes. Pharm Res 2008;25:2097-116.

22. Garg P, Bansal M, Garg M, Arora B. Creating awareness about the painless nature of early breast cancer lump is important in low-income countries. Breast J 2010;16:101-2.

23. Odusanya O०, Tayo OO. Breast cancer knowledge, attitudes and practice among nurses in Lagos, Nigeria. Acta Oncol 2001;40: 844-8.

24. Oluwatosin OA, Oladepo O. Knowledge of breast cancer and its early detection measures among rural women in Akinyele Local Government Area, Ibadan, Nigeria. BMC Cancer 2006;6:271.

25. Magrath I, Bey P, Shad A, Sutcliffe S. Cancer funding in developing countries: the next health-care crisis? Lancet 2010;376:1827.

26. Aweke YH, Ayanto SY, Ersado TL. Knowledge, attitude and practice for cervical cancer prevention and control among women of childbearing age in Hossana Town, Hadiya zone, Southern Ethiopia: community-based cross-sectional study. PLoS One 2017;12:e0181415.

27. Haileselassie W, Kaba M, ArayaSellasie M, Mulugeta T, Lako Labisso W. Challenges and opportunities in cancer diagnosis in Ethiopia: in-depth exploration of practitioners' view. Int J Curr Res 2017;9:54662-8.

28. Mwaka AD, Orach CG, Were EM, Lyratzopoulos G, Wabinga H, Roland M. Awareness of cervical cancer risk factors and symptoms: cross-sectional community survey in post-conflict north- ern Uganda. Health Expect 2016;19:854-67.

29. Ekechi C, Olaitan A, Ellis R, Koris J, Amajuoyi A, Marlow LA. Knowledge of cervical cancer and attendance at cervical cancer screening: a survey of Black women in London. BMC Public Health 2014;14:1096.

30. Maranga IO, Hampson L, Oliver AW, Gamal A, Gichangi P, Opiyo A, et al. Analysis of factors contributing to the low survival of cervical cancer patients undergoing radiotherapy in Kenya. PLoS One 2013;8:e78411.

31. Vanderpuye VDNK, Olopade OI, Huo D. Pilot survey of breast cancer management in sub-Saharan Africa. J Glob Oncol 2016;3:194-200.

32. International Agency for Research on Cancer. Section of cancer surveillance. https://www.iarc.fr/research-sections_trashed/research- sections-csu/. Accessed January 12, 2015.

33. Beaglehole R, Yach D. Globalisation and the prevention and control of non-communicable disease: the neglected chronic diseases of adults. Lancet 2003;362:903-8.

34. DeSantis CE, Bray F, Ferlay J, Lortet-Tieulent J, Anderson BO, Jemal A. International variation in female breast cancer incidence and mortality rates. Cancer Epidemiol Biomarkers Prev 2015;24:1495-506.

35. Sharma V, Kerr SH, Kawar Z, Kerr DJ. Challenges of cancer control in developing countries: current status and future perspective. Future Oncol 2011;7:1213-22.

36. Randall TC, Ghebre R. Challenges in prevention and care delivery for women with cervical cancer in sub-Saharan Africa. Front Oncol 2016;6:160.

37. International Atomic Energy Agency. Division of Public Information. A silent crisis: cancer treatment in developing countries. Vienna, International Atomic Energy Agency Austria, 2003.

38. International Atomic Energy Agency (IAEA). IAEA human health series: planning national radiotherapy services: a practical tool. Vienna, IAEA, 2010.

39. Abdel-Wahab M, Bourque JM, Pynda Y, Iżewska J, Van der Merwe D, Zubizarreta E, et al. Status of radiotherapy resources in Africa: an International Atomic Energy Agency analysis. Lancet Oncol 2013; 14:e168-75.

40. Gopal S, Wood WA, Lee SJ, Shea TC, Naresh KN, Kazembe PN, et al. Meeting the challenge of hematologic malignancies in sub-Saharan Africa. Blood 2012;119:5078-87.

41. Yohana E, Kamuhabwa A, Mujinja P. Availability and affordability of anticancer medicines at the Ocean Road Cancer Institute in Dar es Salaam, Tanzania. East Afr J Public Health 2011;8:52-7.

42. Baile WF. Communication competency in oncology: legal, ethical and humanistic imperatives. Psycho-Oncol 2006;15:S6.

43. Suleiman DE. Pathologist-clinician collaboration: a marriage of necessity toward improving the quality of patient care. Ann Niger Med 2015:9:1-3.

44. Norum J, Grev A, Moen MA, Balteskard L, Holthe K. Information and communication technology (ICT) in oncology. Patients' and relatives' experiences and suggestions. Support Care Cancer 2003;11:286-93.

45. Kwankam SY, Ningo NN. Information technology in Africa: a proactive approach and the prospects of leapfrogging decades in the development process. Accessed January 3, 2016. 